\title{
Enfin un gène pour le syndrome de Rett
}

A A conn de cest dermitere années, alors que de plus en plus de gènes impliqués dans des maladies humaines étaient découverts, le syndrome de Rett (RTT) restait une énigme, embarrassante pour les chercheurs, douloureuse pour les familles de malades (m/s 1996, $n^{\circ} 11$, p. 1280).

Il faut reconnaître que la tâche n'était pas facile. Cette encéphalopathie progressive, qui ne touche presque exclusivement que les filles, est sporadique dans plus de $99 \%$ des cas. Les enfants atteintes semblent normales à la naissance et au cours des premiers mois de vie. Vers 18 mois apparaît une régression, parfois rapide, des premières acquisitions, puis un profond retard mental ainsi qu'une ataxie s'installent progressivement. En l'absence de tout signe biologique, des critères cliniques d'inclusion très stricts ont été définis pour asseoir le diagnostic: retard de développement de -2DS du périmètre crânien (généralement normal à la naissance), perte des mouvements volontaires des mains avec enlacements des doigts et accès d'hyperpnée, entre autres.

Que le syndrome de Rett soit génétique, personne n'en doutait, mais l'impossibilité d'effectuer des analyses de ségrégation, faute de familles multiplex, a d'abord conduit à proposer de nombreux modèles. Le plus simple était évidemment la survenue d'une mutation dans un gène dominant lié à l'X, létal chez le garçon. Contrairement aux autres maladies de ce type, aucune diminution du nombre des garçons dans les familles à RTT n'est observée. Il fallait donc admettre l'hypothèse d'une fréquence très élevée de mutations, puisque l'incidence du syndrome de Rett est d'environ 1 sur 10000 à 15000 naissances de filles.

De nombreuses et laborieuses analyses de l'inactivation de l'X chez les filles RTT et leurs mères furent réalisées sans résultat concluant. Devant l'impossibilité de parvenir à trouver un locus, les études comparatives des haplotypes de quelques sœurs et demi-sours RTT existant dans le monde finirent par donner une carte d'exclusion de l'X qui épargnait la région $\mathrm{Xq} 28$ [1]. Celle-ci devenait alors une région d'intérêt que de nombreuses équipes se mirent à explorer, sachant que la découverte du gène en cause aurait, comme pour la maladie de l'X fragile, des implications dépassant largement le cadre de cette maladie.

Des chercheurs américains (Houston TX, Stanford, CA, USA), qui travaillaient depuis longtemps sur le syndrome de Rett et la région Xq28 viennent enfin de découvrir un gène dont les mutations sont indiscutablement responsables de cas de RTT [2].

Il s'agit du gène $M E C P 2$ qui code pour une protéine MeCP2 identifiée en 1992 en raison de sa propriété de se lier à l'ADN méthylé. Elle se lie sélectivement aux résidus 5-méthylcytosine des dinucléotides CpG répartis dans tout le génome des mammifères. Ces résidus sont localisés de préférence dans les régions promotrices des gènes soumis à la répression transcriptionnelle après méthylation. MeCP2 contient deux domaines fonctionnels, un domaine MBD (methyl CpG binding domain) nécessaire pour la liaison à la 5méthylcytosine et un domaine de répression transcriptionnelle (TRD) qui interagit avec une histone désacétylase et le co-répresseur transcriptionnel SIN3A [3]. Cette interaction entraîne une désacétylation des his- tones et un blocage de la transcription [4].

Dans cinq cas sporadiques sur 21 analysés, des mutations dans la région codante touchant l'un et l'autre domaine ont été observées. MeCP2 possède une longue région 3 ' non transcrite dont certaines séquences sont très conservées au cours de l'évolution et qui doit jouer un rôle dans la régulation post-transcriptionnelle de MeCP2. [5]. Cette région 3' UTR mériterait d'être aussi explorée dans les autres cas de syndrome de Rett. Dans les quelques cas familiaux, l'absence de mutation chez les parents (en particulier chez une mère vectrice obligatoire) suggère l'éventualité d'une mosaïque germinale et, d'une façon plus générale, une fréquence élevée des mutations.

Dans cette maladie touchant exclusivement les filles, la découverte de la perte de fonction d'un facteur intervenant dans la régulation épigénétique a de quoi séduire. C'est la première fois qu'un tel gène est impliqué dans une maladie humaine. D'autres gènes semblables mais autosomiques (en particulier les gènes codant pour des répresseurs à domaine MBD [6]) peuvent être impliqués dans d'autres retards mentaux non syndromiques [7].

Reste à comprendre comment agit ce répresseur transcriptionnel ubiquitaire pour entraîner une atteinte aussi spécifique du système nerveux. On sait que les disomies fonctionnelles de l'X (par perte du XIST dans des chromosomes $X$ délétés, par exemple) ont un retentissement très sévère sur le phénotype, et spécialement sur le développement psycho-moteur $\left(\mathrm{m} / \mathrm{s} 1990, n^{\circ} 5, p .481\right.$ et 1995, $n^{\circ} 8$, p. 1188). Parmi beaucoup d'autres, une hypothèse peut être 
proposée: la région 3' UTR de MeCP2 est dotée d'une polyadénylation alternative, avec une variété de transcrits dont certains sont fortement exprimés dans le cerveau. La perte de fonction d'une de ces protéines dans les neurones post-mitotiques pourrait entraîner une surexpression de gène(s) qui serait dommageable pour la maturation $\mathrm{du}$ système nerveux. L'invalidation du gène Mecp 2 chez la souris montre le caractère létal de la perte du gène chez les mâles [8]. La création d'une lignée conditionnelle, où Mecp2 serait spécifiquement invalidé dans le cerveau, serait un bon modèle pour l'étude du syndrome de Rett.

\section{RÉFÉRENCES}

1. Siriani N, Naidu S, Pereira J, Pillotto RF, Hoffman EP. Rett syndrome confirmation of X-linked dominant inheritance, and localisation of the gene to Xq28. Am J Hum Genet 1998; 393: 1552-8.

2. Amir RE, Van den Veyver IB, Wan M, Tran CQ, Francke U, Zoghbi HY. Rett syndrome is caused by mutations in X-linked $M E C P 2$, encoding methyl-CpG- binding protein 2. Nat Genet 1999; 23: 185-8.

3. Jones PL, Veenstra GJC, Wade PA, et al. Methylated DNA and MeCP2 recruit histone deacetylase to repress transcription. Nat Genet 1998; 19: 187-91.

4. Taddei A, Almouzni G. Les acétyl-transférases et désacétylases des histones: des corégulateurs de la transcription. Med Sci 1997 ; 13: 1205-11.

5. Coy JF, Sedlacek Z, Bächner D, Delius H, Poustka A. A complex pattern of evolutionary conservation and alternative polyadenylation within the long 3'-untranslated region of the methyl-CpG-binding protein 2 gene (MeCP2) suggests a regulatory role in gene expression. Hum Mol Genet 1999; 8: 1253-62. 6. Hendrich B, Abbott C, McQueen H, Chambers D, Cross S, Bird A. Genomic structure and genomic mapping of the murine and human $m b d 1, m b d 2, m b d 3, m b d 4$ genes. Mamm Genome 1999; 10: 906-12.

7. Willard HF, Hendrich BD. Breaking the silence in Rett syndrome. Nat Genet 1999; $23: 127-8$.

8. Tate P, Skarnes W, Bird A. The methylCpG binding protein MeCP2 is essential for embryonic development in the mouse. Nat Genet 1996 ; 12 : 205-8.

\section{Simone Gilgenkrantz}

9, rue Basse, 54330 Clerey-sur-Brenon, France. 